\title{
Article \\ Developing a BIM-Based MUVR Treadmill System for Architectural Design Review and Collaboration
}

\author{
Calvin Chung Wai Keung (D), Jung In Kim * and Qiao Min Ong \\ Department of Architecture and Civil Engineering, City University of Hong Kong, Hong Kong, China; \\ cwkeung@cityu.edu.hk (C.C.W.K.); qmong2-c@my.cityu.edu.hk (Q.M.O.) \\ * Correspondence: jungikim@cityu.edu.hk
}

Citation: Keung, C.C.W.; Kim, J.I.; Ong, Q.M. Developing a BIM-Based MUVR Treadmill System for Architectural Design Review and Collaboration. Appl. Sci. 2021, 11, 6881. https://doi.org/10.3390/ app11156881

Academic Editor: Enrico Vezzetti

Received: 30 June 2021

Accepted: 23 July 2021

Published: 27 July 2021

Publisher's Note: MDPI stays neutral with regard to jurisdictional claims in published maps and institutional affiliations.

Copyright: (c) 2021 by the authors. Licensee MDPI, Basel, Switzerland. This article is an open access article distributed under the terms and conditions of the Creative Commons Attribution (CC BY) license (https:// creativecommons.org/licenses/by/ $4.0 /)$.

\begin{abstract}
Virtual reality (VR) is quickly becoming the medium of choice for various architecture, engineering, and construction applications, such as design visualization, construction planning, and safety training. In particular, this technology offers an immersive experience to enhance the way architects review their design with team members. Traditionally, VR has used a desktop PC or workstation setup inside a room, yielding the risk of two users bump into each other while using multiuser VR (MUVR) applications. MUVR offers shared experiences that disrupt the conventional single-user VR setup, where multiple users can communicate and interact in the same virtual space, providing more realistic scenarios for architects in the design stage. However, this shared virtual environment introduces challenges regarding limited human locomotion and interactions, due to physical constraints of normal room spaces. This study thus presented a system framework that integrates MUVR applications into omnidirectional treadmills. The treadmills allow users an immersive walking experience in the simulated environment, without space constraints or hurt potentialities. A prototype was set up and tested in several scenarios by practitioners and students. The validated MUVR treadmill system aims to promote high-level immersion in architectural design review and collaboration.
\end{abstract}

Keywords: virtual reality (VR); multiuser VR (MUVR); omnidirectional treadmills; architectural design review; design collaboration

\section{Introduction}

The advanced building information modeling (BIM) technology has completely changed how architects approach building design. The potent BIM tools energize architects' innovation to create exceptional designs that could not be made by most traditional methods [1]. However, it is challenging for architects to present complicated and sophisticated designs if conventional 2D or 3D representations are adopted. Similarly, the ineffective design presentation might result in communication errors among team members [2]; the consequence is often costly design variations in the construction stage [3]. Thus, virtual reality (VR) has much potential for architects and designers, as this revolutionary technology transports users into an immersive virtual environment, allowing them to explore a simulated representation of a particular room, floor, or building design as a whole with their partners. VR offers practical solutions with computer-generated artificial realities, and it is now entering mainstream use in various fields, such as entertainment, education, design, engineering, and health services [4].

VR popularity in the architecture, engineering, and construction (AEC) industries is increasing as it becomes more affordable for end-users [5,6]. Using VR as a platform for content delivery allows better user engagement than other traditional methods, as VR headsets remove external distractions by shutting out the physical world, creating a complete immersion experience. Most AEC researches mainly leverage the visualization function offered by BIM-based VR applications to support design and construction activities [7-10]. 
For instance, BIM design can be monitored and compared through VR applications for decision-making and simulated for pre-construction planning. On the other side, VR is also helpful in dealing with misunderstanding, spatial sense issues, and clash-detection problems in the design review process [11]. Thus, it offers considerable potentialities for improving the inefficiencies in current $2 \mathrm{D}$ and $3 \mathrm{D}$ design review and coordination practice. In addition, interactive multiuser VR (MUVR) has been long established in construction researches, such as safety training. MUVR has been proven beneficial to the overall site safety performance [12]. However, current BIM-based VR applications in architectural design are still limited to single-user usages and lack of interactions among users. Collaborative $\mathrm{VR}$ allows multiple users to attend virtual design or presentation sessions in the $3 \mathrm{D}$ environment, and they can work collaboratively in the same room or remotely from different locations [5].

There is also a common problem seldom addressed by researchers or practitioners when using VR devices in architectural applications. VR users are usually facing locomotion limitations due to the designated space constraint [13]. The limits of the real environment are identified by a virtual grid fence that avoids VR users bump into actual physical walls or objects in the perceived environment. This constraint affects the navigation efficiency of 3D models for buildings with considerable floor areas (e.g., airports, exhibition halls, and shopping arcades) and leads to the risk of two users bump each other when using MUVR applications. Although artificial VR locomotion (e.g., teleportation) is one of the solutions for users to navigate the virtual environment by moving themselves to different locations with the use of hand-held controllers, this method tends to cause VR sickness due to the discrepancy between expected and perceived sensory information [14]. As such, omnidirectional treadmills are the cutting-edge solutions for walking simulations in the virtual environment. The treadmills enable users to move freely, without the concern of navigational space limitations. Thus, they enhance the illusion of unconstrained human movements and make the overall VR experience more realistic and immersive [13]. Such an immersive walking experience can increase users' sense of presence and motivate users to interact naturally with others in the virtual environment.

Given these points, this study aimed to develop a BIM-based MUVR treadmill system to promote high-level immersion in the design review process, offering architects a more realistic way to design BIM models collaboratively in the virtual environment. A prototype was set up by integrating MUVR applications into omnidirectional treadmills based on the client-server model. The system can support multiuser interactions without motion constraints in the virtual environment. Ultimately, architects are encouraged to collaborate virtually with their team members during the design review process. The system was validated by participants who tested the prototype and provided feedback on system design, particularly when problems or challenges were identified. The participants also offered objective evidence to ensure that the system aligns with the professional practice and the VR model is a practical and complete representation of architectural characteristics. Further, as the level of interest and appeal in VR technology is different with human age, the participants were divided into two groups to detect any unconscious bias arising from users' technology readiness. Finally, discussions of research findings and recommendations for the system improvements are presented.

\section{Theoretical Background}

Researchers and practitioners have developed VR technology since the early 1990s. VR is a computer-generated environment with senses and objects that appear to be real. Thus, VR technology can be adopted as a communication system component that creates a simulated space and embeds humans as an integral part of the system [15]. Based on virtual displays supported by unique processing systems and non-visual sensory components, such as tactile and auditory functions, VR users can experience a truly immersive-simulated virtual environment [16]. The terminology of VR can be characterized by the 5Is: intensive, interactive, immersive, illustrative, and intuitive [17]. Such characteristics have created 
ample research and application opportunities in various fields, such as automation, healthcare, and construction. Their limitations for spatial or temporal inaccessibility to realistic objects develop the need and ability to realize certain levels of accuracy in the virtual setting [10]. Moreover, space and equipment functionalities can be substantially validated through additional interaction capabilities in the virtual environment [18], achieving high efficiencies in the design review process. However, some studies proved that youths enjoy new technologies (e.g., VR applications) significantly more than adults do and had a higher willingness to adopt new technological products $[19,20]$. This situation can be explained by the concept of technology readiness, which refers to people's propensity to use new technologies to achieve goals in home life and at work [21].

In the AEC industry, the extensive BIM development brings VR applications to a higher level. The integration of BIM into VR optimizes the performance as BIM-based models contain rich information that serves as a reliable basis to facilitate decision-making during the whole project life cycle, starting from planning, design, and construction to operation and maintenance stages [22]. In particular, VR has predominantly been applied in architectural design review. The incentive of its widespread adoption is the significant visual improvement arising from virtual models developed by gaming engines [10]. Architects and designers can review the design in a 1:1 scale immersive virtual environment to analyze design factors such as material types and dimensions to develop an effective building design [23]. In this highly immersive and realistic way, VR applications can judge the realistic visual impacts and technical aspects [7]. For instance, architects can better figure out the spatial relationships between building components to resolve design conflicts, check headroom compliances, and verify the required assembly detailing of the final products [24,25]. Furthermore, VR can be adopted to produce virtual mock-ups replacing physical building models for design overviews [18]. This efficient approach enables stakeholders to consider and compare different design options simultaneously without much cost and time implication [26].

BIM-based VR applications have triggered a significant paradigm shift in the AEC industry. Construction professionals capitalize on integrating BIM into VR technology to enhance problem-solving efficiency in an immersive virtual environment. In addition, the advent of cutting-edge VR applications provides opportunities for immersive visualization that can help construction professionals to improve the sense of scale and spatial relationships of architectural and engineering design [7]. However, some challenges of using VR have been identified, such as the capability of running high-demand BIM software in VR applications, the support of multiuser applications, and the human locomotion for VR manipulations.

Construction professionals come from various disciplines, so an interdisciplinary approach is vital to address today's complex engineering and construction problems. As such, the concept of MUVR disrupts the limitations of the conventional single-user VR setting where VR has used a gaming PC, yielding challenges when running the latest construction applications. The typical MUVR platform is set up by using a client-server model [27-29]. Unlike traditional single-user VR applications, MUVR platforms are established to enable real-time interactions and communications between multiple stakeholders in a shared virtual environment. A server is integrated to manage over system displays, and each client can access a data copy to be displayed, either on a local drive by the local area network or a shared network drive by the wide area network. Once a client is connected to the server, the server can update the display and synchronize the client's view regularly. On the other side, each client transmits pose information from its headsets and controllers to the server, relayed to other clients for displays. This framework also supports different modes that enable users to read particular results individually without sharing them with other clients. This setup of MUVR platforms effectively supports real-time interactions between users in the shared virtual environment [27].

In most current VR applications, users are only limited to moving in the predetermined physical space, which may constrain the natural interaction in the virtual environment. 
The gaming treadmills can be an option to offer user movement ability in the virtual environment by fixing users on a stationary point and reducing their foot friction, so that they can walk in a stationary platform naturally [30]. VR treadmills have been popularly adopted in the E-sport entertainment and healthcare industry [31,32]. The self-paced VR treadmills can be bowl-shaped or flat-shaped, and their integration into VR applications can provide users with an immersive walking experience [13]. Moreover, VR treadmills provide great potential in becoming a safe simulator that enables active human locomotion in the virtual environment. For instance, the omnidirectional treadmill is a VR platform designed to bring the user back to the center of the active surface when he moves in any direction and steps off the active surface, avoiding walking over the edge of the platform [33]. Human locomotion and usability in the virtual environment are the critical aspects of the omnidirectional treadmill [34]. This kind of VR treadmill offers benefits such as allowing users to run and jump naturally on a designated platform during VR simulation, as every foot movement is tracked by the hardware. The average enjoyment level during physical activities in VR on treadmills is higher, because this movement can increase user participation in the virtual environment [35]. Thus, there is great potential to adopt VR treadmills in the AEC industry to enhance the users' immersive experience, as the current VR applications are limited to the conventional way without human locomotion. Significantly, the setup of multiple treadmills can avoid real-world collisions between users operating MUVR platforms in the same shared physical room.

\section{System Framework}

This study proposed a BIM-based MUVR treadmill system to offer high-level immersion in design review and collaboration in the design stage. Figure 1 displays the system framework that consists of four core modules: (1) BIM model, (2) VR platform with interactive functions, (3) VR headsets and treadmills, and (4) MUVR server. It is computationally intensive if starting with a building model built by BIM software because many hidden faces and duplicating vertices are contained in BIM models. Thus, before importing a BIM model into the VR platform, the BIM model should be optimized by the computer graphic program's previsualization process. In other words, the BIM model needs to be fully optimized so that it can become computationally light-weighted with low poly-geometry, minimum overlapping faces and vertices, and minimum repetitive textures to reduce the file size and ensure smooth VR interactions without clashes. After the geometric optimization, the BIM model is imported into the VR platform to become the VR model. Several criteria must be fulfilled to establish a realistic virtual environment, including the real-world immersive experience, realistic object texture, and lighting effect. The VR model is further optimized for the lighting performance, such as prebaked lighting effects (e.g., normal map or ambient occlusion map) to produce the surrealistic rendering quality. Then, a pair of VR headsets and hand-held controllers are adopted for virtual displays and system manipulations, respectively. Regarding human locomotion, a pair of omnidirectional treadmills are integrated into the VR platform to offer users the walkthrough capability in the virtual environment without physical space constraints. All these devices are mutually supported and compatible with the VR platform. Finally, a synchronization system was developed to support multiuser interactions in the virtual environment. The system adopts the client-server model to facilitate multiuser interactions. As such, an MUVR server was provided to connect the network of other workstations (i.e., users) to access, hold, and distribute the requested information to clients over a local area network. Due to its high processing power and sufficient random-access memory in managing the massive load, the server takes a vital role in optimizing the synchronization performance between multiusers, offering users stable and reliable MUVR experiences. 


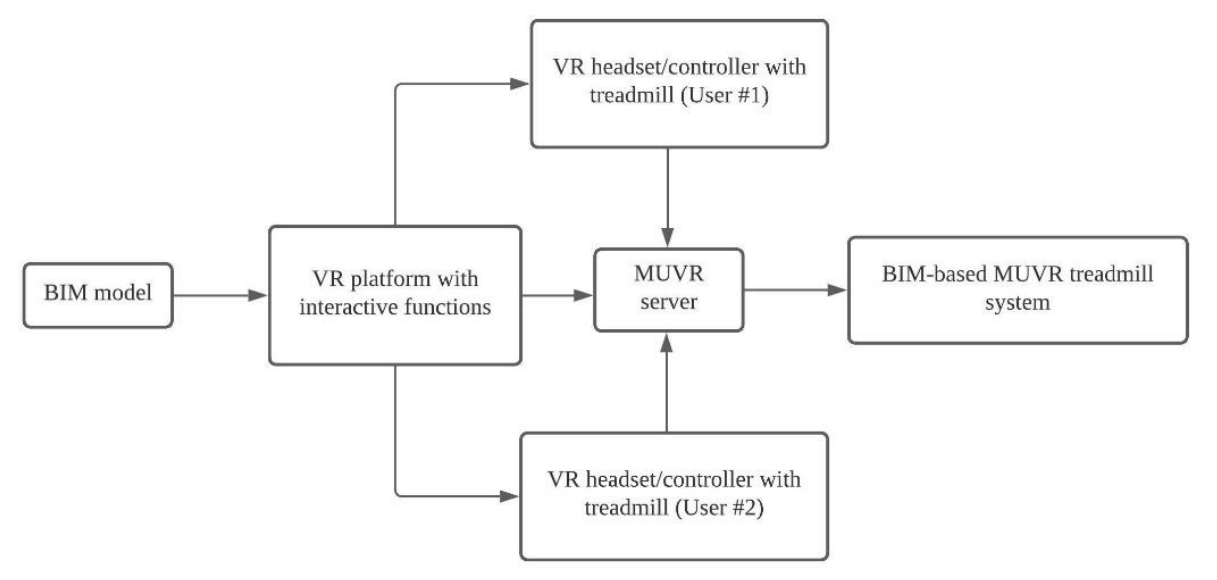

Figure 1. Framework of the BIM-based MUVR omnidirectional treadmill system.

Four interactive functions were developed by intensive coding and programming to enhance architectural design review and collaboration effectiveness. The functions are tailor-made features that facilitate architects to review the design with their team members interactively. The first function is the primitive creation with object manipulations. This tool allows different objects with standard shapes, such as cubes, rectangles, spheres, and arrows, to be created in any dimension in the virtual environment. The objects created can be relocated anywhere or any direction by users without limitations. This tool aims to unlock user creativity during the design review process. Architects can also review different design options with their team members, making decisions efficiently and less costly. The second function is the freeform annotation tool. Annotations can be created on any object or even in the air of the virtual environment by pen-style mark-ups. Different brush sizes and pen colors can be chosen to perform better notetaking and sketching effects when discussing design detailing. These functions can be done by employing the line renderer component of the software to create lines synchronized in real-time between multiusers. The third function is the measurement tool. This tool offers quick quantity takeoff in the virtual environment, and a virtual indicating line is provided so that the starting point can be more accurate. This kind of freestyle measurement (i.e., in any direction, $x, y$, or $\mathrm{z}$ ) would not be possible in any 2D or 3D authoring software. The tool can be applied for resolving design conflicts by determining spacing distances and minimum sizes, such as pipework positions and lift door openings. The last function is the scale-model mode for small-scale views of models for design overview. The scale-model mode is another way of navigation other than normal building walkthroughs in the virtual environment. This function allows users to visualize the whole building like a physical model to understand better the overall design for strategic analysis and design overview. The scale-model mode also allows users to visualize any particular section in a closer and detailed inspection. For example, users can utilize the scale-model mode to modify skylights or even measure whole building heights that would not be possible in real-world situations. The scaling function further enables users to review the virtual environment in different scales by zooming in or out. Figure 2 shows the interactive functions of the system and illustrates how they are manipulated in the virtual environment. 


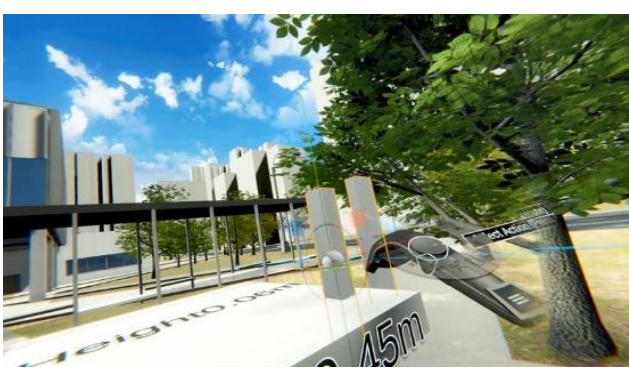

(a)

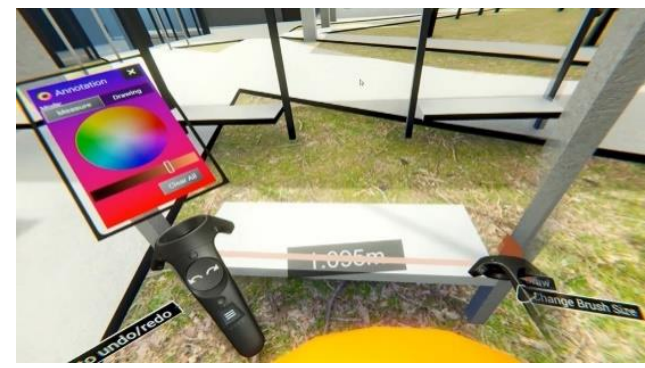

(c)

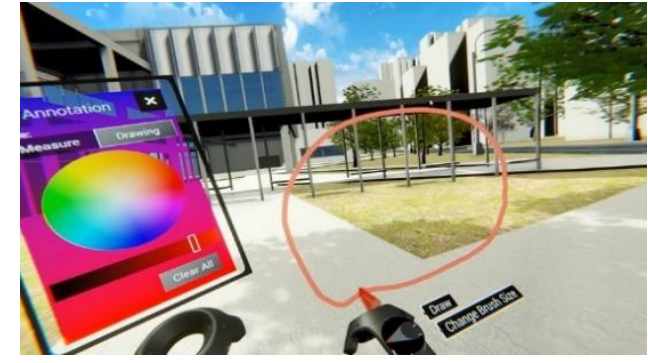

(b)

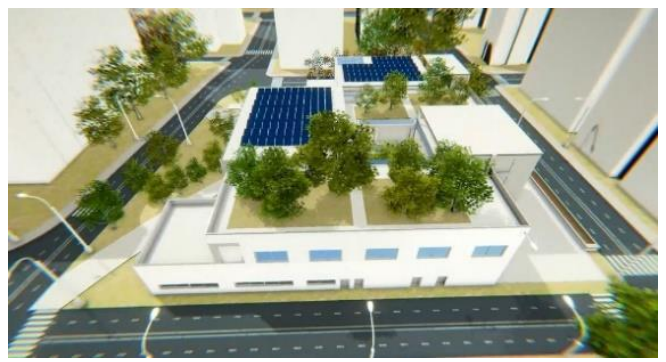

(d)

Figure 2. Interactive functions of the system: (a) primitive creation, (b) annotation, (c) measurement, and (d) scale-model mode.

\section{System Development}

\subsection{Software Requirements}

Various software programs were chosen to develop the BIM-based MUVR treadmill system. For instance, the tools for BIM modeling, BIM model optimizations, VR platform development, and MUVR network establishment were used. For the BIM model authoring tool, Autodesk Revit was adopted because of its high accuracy and efficiency in design and documentation across the project lifecycle. Revit is one of the dominant BIM solutions for architectural design in the international commercial and research markets [36-38]. The critical difference between Revit and other modeling software is that Revit comes with a single package that includes modeling of building structures and mechanical, electrical, and plumbing (MEP) components. This advantage brings conveniences to other engineering disciplines for further BIM model development.

To integrate BIM models into VR applications, BIM models are produced in their native format based on the predetermined project requirements and then exported into a Filmbox (FBX) file, a flexible file format for geometric optimizations before importing into VR platforms. The FBX file is a means of importing 3D models into gaming or animation packages because it can keep most of the geometry information, material data, light, and environmental settings in the original BIM model. Autodesk 3Ds Max was chosen as the 3D computer graphics program due to its widespread adoption of geometric optimizations for design visualization and animation [37,39]. In the computer graphic environment, each 3D object consists of polygons which can be further be subdivided into faces. However, these faces are often hidden and are not displayed in the viewport by default. After importing the FBX-based BIM model into the Autodesk 3Ds Max, the alleged vertices, edges, polygons, and faces of each 3D object were translated into the editable poly and shown in the $\mathrm{xView}$. The editable poly modifier was applied to optimize the poly-geometry BIM objects to reduce the model's file size with minimum overlapping edges, vertices, and repetitive textures. There are three primary modifiers in 3Ds Max to reduce the polygon count of 3D models: Optimize modifier, ProOptimizer modifier, and MultiRes modifier. These modifiers aim to reduce the model processing complexity during the MUVR interaction by lowering the overall file size while maintaining BIM models' shape and visual quality.

The VR platform was developed by using the gaming engine, because it can power VR applications with a high frame rate and realistic user experience in the virtual environ- 
ment [30]. Unity was chosen as the development tool because it offers a more user-friendly user interface and greater cross-platform, posing considerable potential for further applications and developments. Furthermore, Unity enables the creation of complex projects without over-demanding a powerful setup like other gaming engines [40]. This characteristic can relieve the hardware burden, especially for the system with multiusers usages. Unity uses C\# or JavaScript primarily in the programming language, so scripting is more flexible, fast, and efficient [30]. In this study, all VR interactive functions were developed by C\# programming scripts in Unity. Unity was also used for lighting optimizations of the VR model. The Unity High-Definition Render Pipeline (HDRP) was deployed to obtain a realistic rendering quality to optimize the lighting effects and post-processing effects. There are two main parts of graphic optimizations regarding the overall lighting effects and material textures under HDRP. For lighting effects, Unity adopts unified and coherent Physical Light Units in the VR world. These units were set based on real-life light's luminosity expressed in Lux or Lumens. Other advanced settings under the light section include the reflection in HDRP, refraction in HDRP, environment lighting, density volume, light layers, and shadows. For material textures, the new features of HDRP Lit Shader are available under the Material Inspector. HDRP also uses ambient occlusion to provide ambient light onto the 3D object's surface with no surface geometry details. Other parameters, such as base color, opacity, metallic surface, smoothness, and coat mask, were adjusted to fully reflect the natural material texture.

Most importantly, the BIM-based MUVR treadmill system was developed based on the availability of multiplayers and networking solutions. UNet was chosen as the solution because it is a native Unity networking system that enables the development of MUVR platforms. Five key features facilitate multiuser applications in the VR world: Transport Layer, Low-Level Application Programming Interface (LLAPI), High-Level Application Programming Interface (HLAPI), Matchmaker, and Relay Server. Firstly, UNet starts with a high-performance Transport Layer based on the User Datagram Protocol to support different application's compatibility. From this Transport Layer, two APIs were built. LLAPI provides comprehensive controls through a socket line interface. At the same time, HLAPI introduces a secure and straightforward client-server network model. Moreover, Matchmaker services support the elementary functionality in configuring rooms for multiuser applications and assist users to locate each other in the virtual environment. Lastly, Relay Server was applied to solve the connectivity issues behind firewalls to ensure VR users are always connected. Nevertheless, there are still some synchronization issues that need to be overcome in the multiuser mode. For instance, every object in the VR world has its transform properties, including positions, rotations, and scales. However, UNet only supports the synchronization of position and rotation properties. Thus, additional programming by C\# was scripted to allow the synchronization of the object's scale between multiusers.

\subsection{Hardware Requirements}

In this study, two essential VR devices were integrated into the BIM-based MUVR treadmill system: VR headsets and omnidirectional treadmills. A MUVR server was also set up to ensure smooth VR interactions, good visual quality, and stable real-time synchronizations between multiple users, such as resolutions, frame rates, tick rates, and buffering time. Furthermore, two sets of Virtuix Omni VR omnidirectional treadmills were installed, as they can achieve an outstanding balance between speed, comfort level, space demanding, and preciseness [13,31]. Each treadmill set includes four sub-devices: the low-friction motion platform, harness, overshoes, and tracking pods. The concave motion platform with low-friction features plays a prominent role in enabling smooth and immersive walking motions in the virtual environment. The supportive ring and harness provide maximum safety and versatility during rapid and unconstrained movements, such as walking, running, and turning swiftly. 
After comparing with other popular VR headsets in the market, two sets of HTC VIVE Pro were selected for the system, due to the advantages of precise room-scale tracking, vibrant screen colors with immensely high contrast, and realistic immersion [41]. The high-resolution active-matrix organic light-emitting diode (AMOLED) screens with the $90 \mathrm{~Hz}$ refresh rate and $110^{\circ}$ field of view are the primary benefits of offering rich colors, contrasts, and comprehensive views in the VR world. Furthermore, four SteamVR base stations support up to $10 \times 10 \mathrm{~m}$ tracking areas in the physical space. The base station performs its function by flooding the room uniformly with a non-visible light (i.e., infrared pulse) so that the receptors on tracked devices can intercept the light and interpret the user location to the base stations. This feature allows sub-millimeter precise tracking, which is ideal for MUVR interactions in this study.

In addition, the MUVR server takes up the role of the authoritative source of events in the multiplayer virtual environment. It is responsible for receiving and processing the clients' input (e.g., spawning objects or lines) and then transmitting and synchronizing accurate data to the connected clients so that the display is stable and reliable $[27,28]$. Installing the MUVR server reduces the original workstation's communication workloads and processing burdens if it serves both client and server roles [42]. Furthermore, by entering the correct internet protocol (IP) address under the local area network, the communication between clients can be accomplished without direct access to the internet. As a result, the local server can perform its role smoothly with stable and reliable performance $[28,42,43]$. In other words, the VR software can be operated more steadily with the VR hardware with minimum forced termination, freezing, crashing, and time delay during data synchronizations between multiusers.

\subsection{System Architecture}

The BIM-based MUVR treadmill system was developed based on the client-server architecture displayed in Figure 3. Using this approach, the VR graphic client workstations handle the computing-intensive graphics, while the MUVR server handles the synchronization communication between the two client stations. Instead of using one workstation to play both client and server roles, this architecture defines workload distributions of the graphic computation and synchronization communication into different workstations, offering greater expansion potential with more VR client usages in future development.

The networking and synchronization for multiuser usages were tested after establishing the client-server architecture. First, a workstation was used as the MUVR server, and the other two workstations were connected to the server by keying in a particular IP address. Troubleshooting was applied to fix the networking problems, and a local area network connection was finally feasible. Then data synchronizations were tested, and the performance was evaluated by the stability and reliability of the system. During the testing, it was also found that the synchronization for the multiuser functionality did not support object scaling, drawings, object manipulations, measurements, and dimensioning. Thus, additional programming was scripted to create a new real-time synchronization system on top of the original networking system facilitating data transfers between multiusers during their interactions in the shared virtual environment. The testing and troubleshooting of the networking and synchronization ensured that the system could achieve high fidelity of the visual quality while maintaining instant responses to all user interactions with a high refresh rate. 


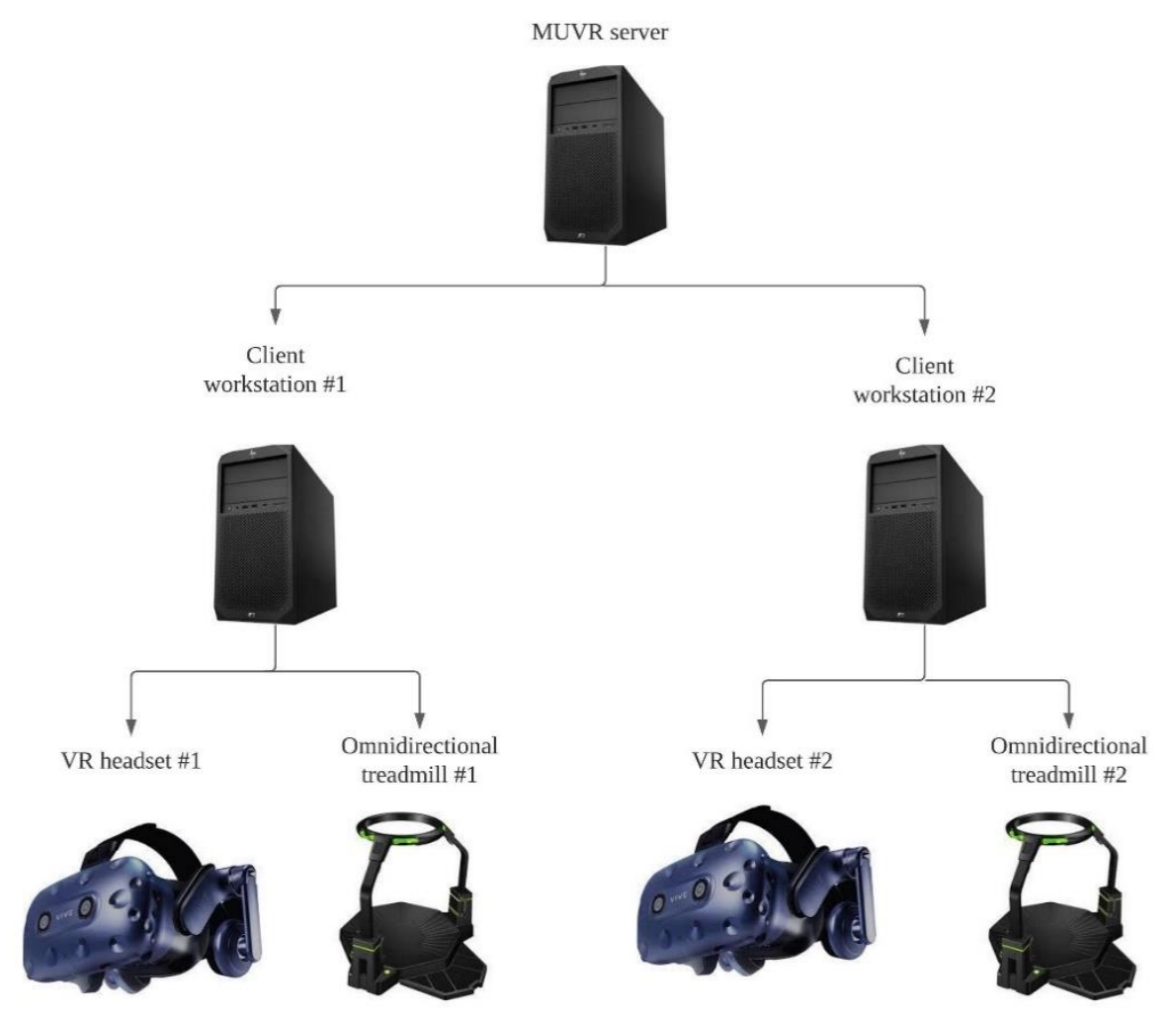

Figure 3. Client-server architecture of the system.

\subsection{System Effectiveness}

A prototype of the BIM-based MUVR treadmill system was set up based on the client-server model. The prototype was tested to validate the system effectiveness in architectural design review and collaboration. The evaluation measures include (1) user interaction, (2) user locomotion, and (3) system synchronization. The system simulates typical design review activities, such as conflict resolution, design detailing, and design overview for the user interaction measures. The evaluation also involves instant design collaboration to test users' creativity. For the measure of user locomotion, the walking experience on VR treadmills was tested. The evaluation includes the preciseness and agility of human locomotion in the shared virtual environment and the sufficiency of safety provisions offered by the treadmills. For the measure of system synchronization, the stability and reliability of the MUVR server were evaluated. These involve the performance of client-server communication in terms of speed and spontaneity.

\section{Validations}

\subsection{Methods}

The target participants of the testing are practicing architects from the AEC industry. Despite that a majority of construction professionals are aware of VR, the levels of their familiarity and enthusiasm of VR technology might be different depending on their age groups. Thus, architecture students studying in local universities were also recruited for the testing. The purposes are to detect any prejudice from user generations against new VR technology and verify whether the research findings of this study vary significantly with different age groups.

Figure 4 shows the BIM-based VR model for testing in this study. The building is a hypothetical community center with a construction floor area of about $2500 \mathrm{~m}^{2}$. It is a three-story building with a total height of $13 \mathrm{~m}$. Before the testing, the participants were given briefings about the research background, building design, client-server architecture, interaction tools, and VR-device operations. Next, the participants were requested to follow the designated routes and accomplish the design review tasks in the virtual environment. 
Afterward, the participants were allowed to navigate the model freely by themselves to test the system thoroughly. The participants were asked to offer valuable feedback on the system's performance and evaluate its effectiveness in design review applications. Finally, the participants were requested to suggest some insightful recommendations for the system improvement.

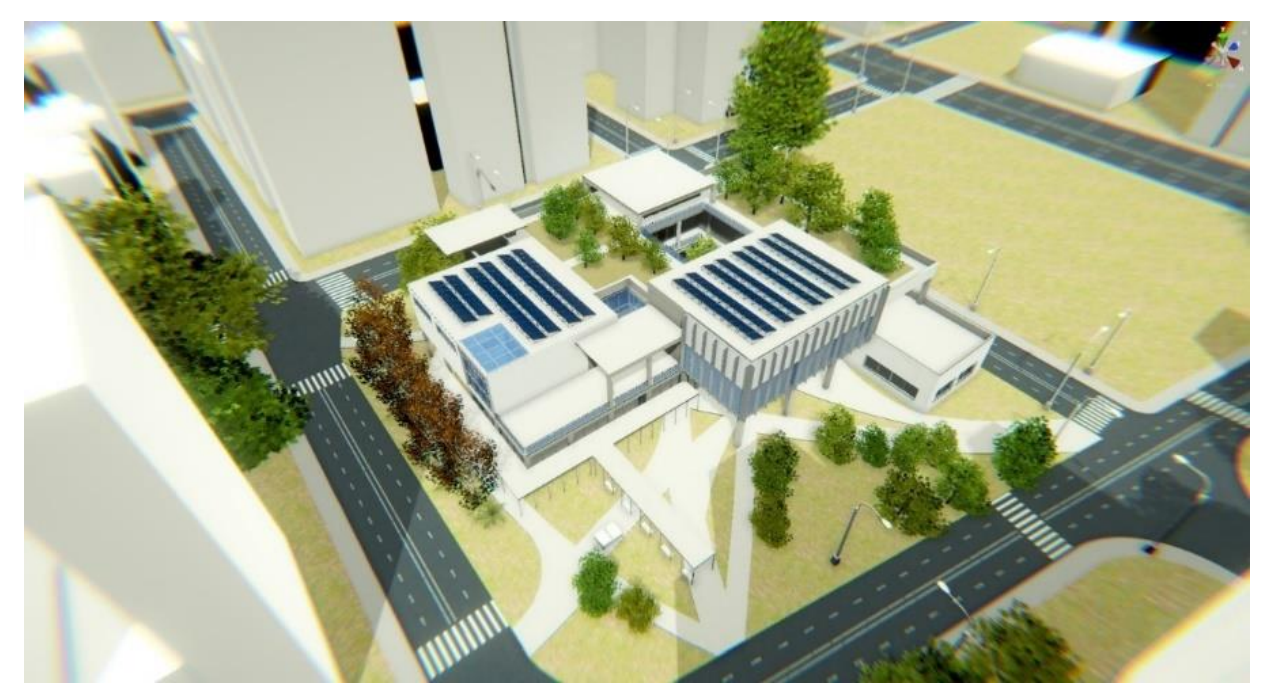

Figure 4. BIM-based VR model.

The testing consisted of several hypothetical tasks carried out by the participants during the design review process. Figure 5 shows the multiple scenarios for the participants to test and verify the interaction tools and VR treadmills. During the testing, the participants accomplished the design review tasks as if they were involved in routine architectural design review with their team members of a building project. The first task is about design conflict resolutions. Starting from the building entrance, the participants walked to the lift doors on the ground floor. One of the participants used the measurement tool to check the height of wall openings. Then the other participants advised the headroom clearance in compliance with the lift installation and applied the annotation tool to highlight the height inadequacy on the openings. A snapshot was finally taken to capture the checking records. The second task is to verify the design details of glass balustrades. The participants went to the corridor on the first floor. One of the participants used the measurement tool to verify the sizes of glazing panels and applied the annotation tool to mark the required assembly details for discussions with the other participant. The third task is the design overview and comparison of the proposed design options. The participants exited the building and adopted the scale-model mode to zoom out the 1:1 building model into a small-scale model. Similar to the overview of a physical model, the participant walked around the model and modified the design by the primitive creation tool. Different options were compared with the consideration of their impacts on the building envelope. The fourth task is about the creation of the conceptual design. The participants kept using the scale-model mode and walked to a vacant site. Using the primitive creation tool, the participants created massing models collaboratively and discussed the creative building models and their effects on the surrounding environments. 


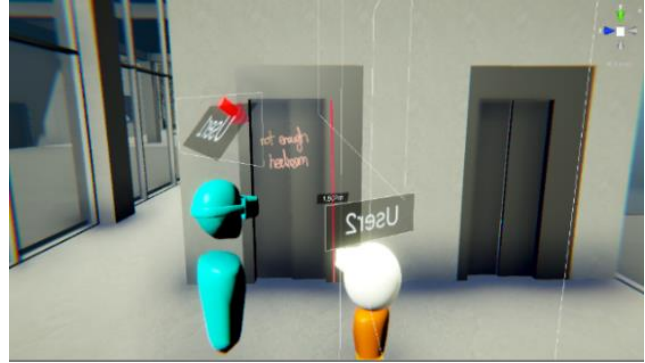

(a)

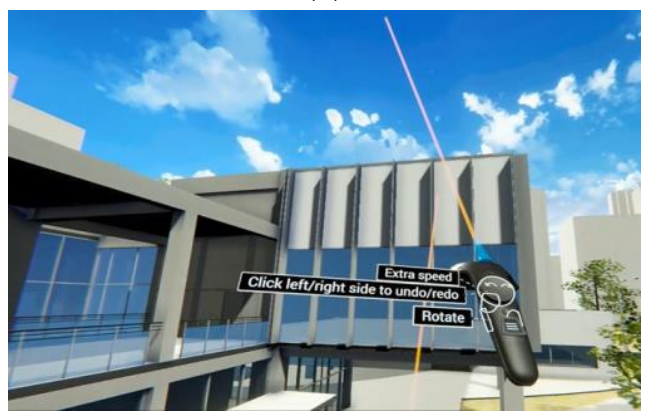

(c)

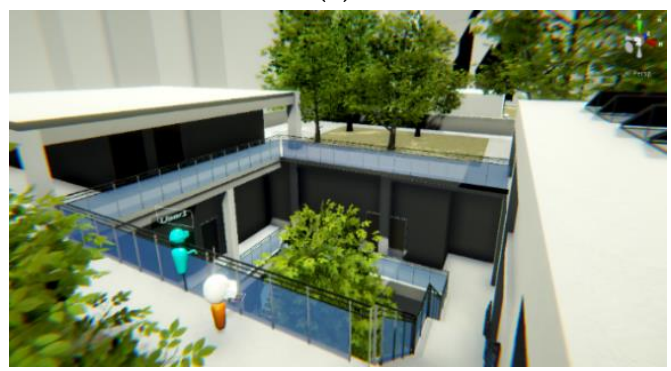

(e)

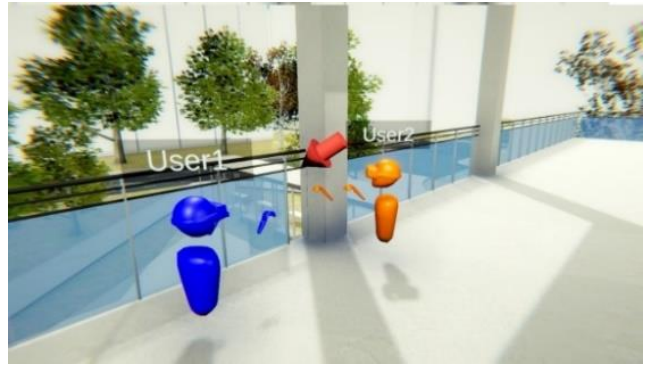

(b)

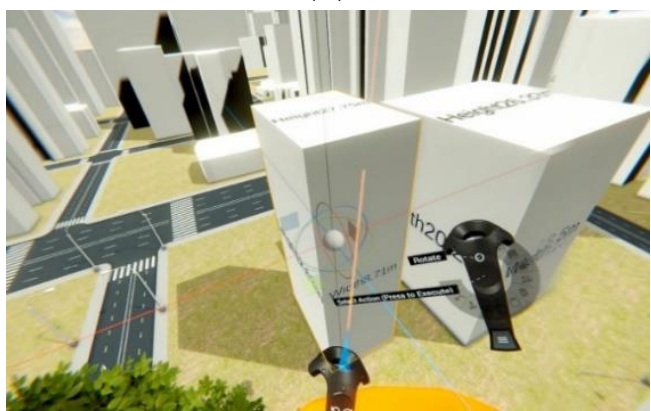

(d)

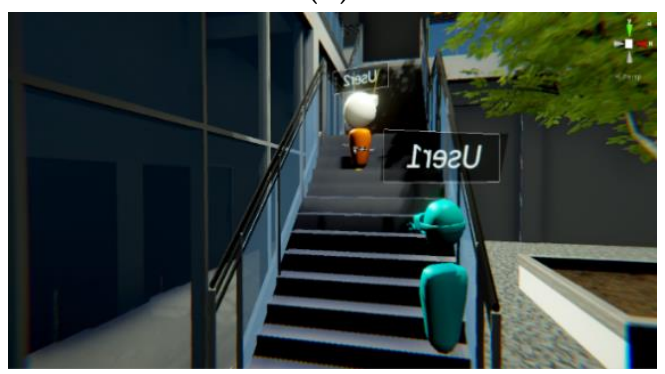

(f)

Figure 5. Multiple scenarios for system testing by a pair of participants: (a) conflict resolution, (b) design detailing, (c) design overview, (d) design creativity, (e) walking along the corridor, and (f) walking along the staircase.

\subsection{Results}

Eight participants agreed to join the testing, and they were divided into two groups. Group 1 consists of four practitioners, while Group 2 includes four students. The practitioners are experienced architects practicing in Hong Kong. They have been involved in various types and sizes of building projects, and their principal job duties are to plan, develop, and implement the architectural design. The students are final-year students studying the undergraduate program of architecture in Hong Kong. They have received basic training in computational design in architecture. All participants have the basic knowledge and know-how of using VR devices but no experience with VR treadmills or similar locomotion platforms. Figure 6 shows a pair of participants testing the system inside the BIM laboratory.

After the testing, all participants were asked to rate their experiences in using the system based on the evaluation measures with a 7-point Likert scale, whereas 7 represents strongly agree, and 1 represents strongly disagree. The evaluation measures were classified into three groups based on multiple scenarios and design review tasks: (1) user interaction, (2) user locomotion, and (3) system synchronization. Mean scores with standard deviations of the evaluation measures were determined. The normality test was also used to determine if the dataset was well modeled by a normal distribution. Then the two-sample $t$-test was conducted to examine whether the opinions of practitioners and students were sufficiently consistent. The mean scores are recognized as having no statistically significant difference 
when the significance level ( $p$-value) is higher than 0.05 [44]. Additionally, all participants were requested to rate the overall immersion level, using the system, according to their experiences of accomplishing the hypothetical tasks and the final stage of free walking. Table 1 summarizes the mean scores and results of the $t$-test.

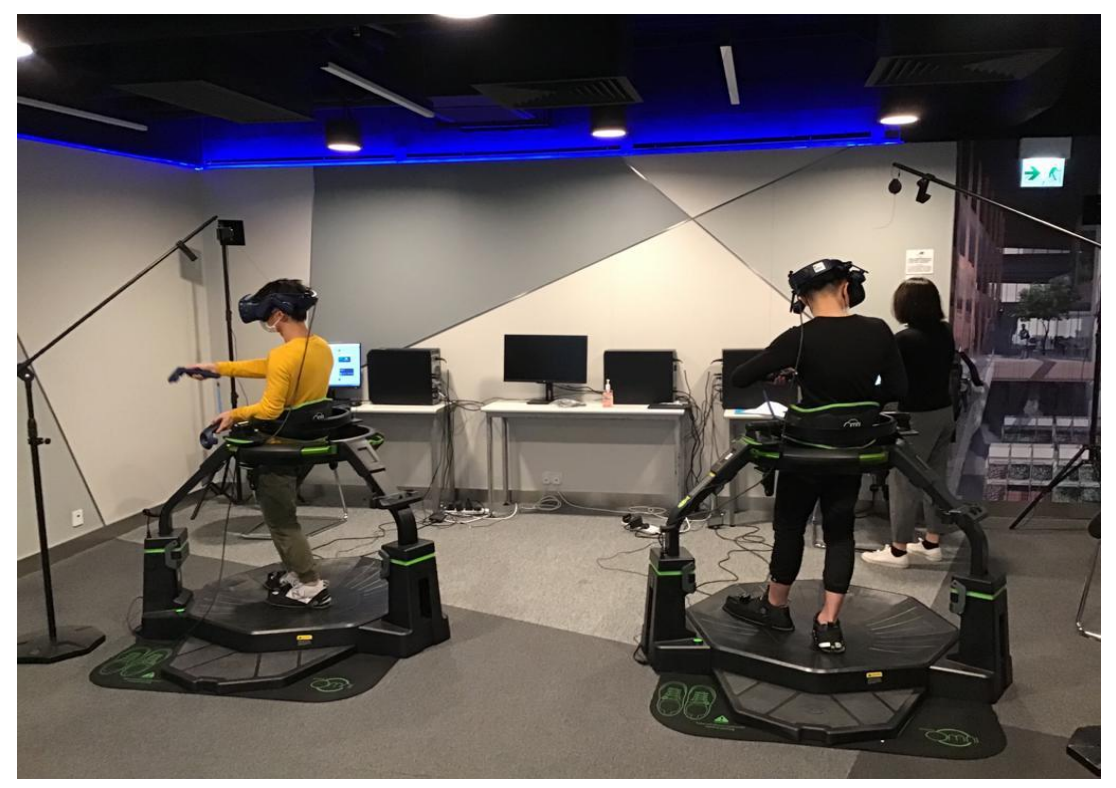

Figure 6. A pair of participants testing the system on MUVR treadmills.

Table 1. Mean scores and results of the $t$-test.

\begin{tabular}{|c|c|c|c|c|c|}
\hline Evaluation Measures & $\begin{array}{c}\text { All } \\
n=8 \\
\text { Mean (SD) }\end{array}$ & $\begin{array}{c}\text { Group } 1 \\
n=4 \\
\text { Mean (SD) }\end{array}$ & $\begin{array}{c}\text { Group } 2 \\
n=4 \\
\text { Mean (SD) }\end{array}$ & $t$ & $\begin{array}{c}\text { Sig. } \\
(p-\text { Value })\end{array}$ \\
\hline \multicolumn{6}{|c|}{ User interaction } \\
\hline Conflict resolution & $5.13(1.45)$ & $5.00(1.87)$ & $5.25(0.83)$ & -0.21 & 0.84 \\
\hline Design detailing & $5.00(1.73)$ & $5.25(1.48)$ & $4.75(1.92)$ & 0.36 & 0.73 \\
\hline Design overview & $5.75(1.20)$ & $5.50(1.66)$ & $6.00(0.00)$ & -0.52 & 0.62 \\
\hline Design creativity & $5.00(1.66)$ & $4.75(1.92)$ & $5.25(1.30)$ & -0.37 & 0.72 \\
\hline \multicolumn{6}{|c|}{ User locomotion } \\
\hline Preciseness & $4.13(1.54)$ & $3.50(1.80)$ & $4.75(0.83)$ & -1.09 & 0.32 \\
\hline Agility & $4.00(1.00)$ & $3.25(0.83)$ & $4.75(0.43)$ & -2.78 & 0.03 \\
\hline Safety & $5.63(1.49)$ & $5.25(1.79)$ & $6.00(1.00)$ & -0.63 & 0.55 \\
\hline \multicolumn{6}{|c|}{ System synchronization } \\
\hline Stability & $5.13(1.69)$ & $4.50(1.80)$ & $5.75(1.30)$ & -0.97 & 0.37 \\
\hline Reliability & $5.63(1.22)$ & $5.00(1.22)$ & $6.25(0.83)$ & -1.46 & 0.19 \\
\hline $\begin{array}{l}\text { Overall immersion } \\
\text { experience }\end{array}$ & $5.50(1.12)$ & $5.00(1.22)$ & $6.00(0.71)$ & -1.22 & 0.27 \\
\hline
\end{tabular}

Group 1: Practitioners. Group 2: Students.

First, the normality test showed that the sampling distribution of the means is normal. Thus, the two-sample $t$-test is valid because the two groups are independent random samples from normal distributions. Second, the means of all participants regarding the interaction experiences are 5 or above, while the measure of design overview received the highest mean (mean $=5.75)$. This measure also received the highest mean in Group 1 -practitioners (mean $=5.5)$ and Group 2-students $($ mean $=6)$. The $t$-test found no statistically significant differences between the two groups of participants in evaluating user interactions. Third, the safety of user locomotion on treadmills was evaluated. This measure had the best performance among all participants (mean $=5.63$ ); the mean of Group 1 is 5.25 , whereas Group 2 is 6 . The results of the $t$-test further show that there 
were no significant differences between the two groups of participants in this measure. However, the measures of preciseness and agility similarly received means below 4 in Group 1 only. In particular, the mean of agility is distinctly low (mean = 3.25). According to the $t$-test, the mean of agility in Group 1 was significantly lower than in Group $2(t=-2.78$, $p=0.03$ ). Fourth, the means of the system synchronization rated by all participants are above 5; especially the measure of reliability showed good performance (mean $=5.63)$. This measure also received the highest mean in Group 1 (mean $=5)$ and Group 2 (mean $=6.25)$. The $t$-test found that the differences between Group 1 and Group 2 in the overall system synchronization were not statistically significant. Finally, all participants were requested to evaluate the overall immersion level provided by the system. Considering the holistic system setup and the user experiences in different scenarios, all participants were satisfied with the overall immersion experience (mean $=5.5)$. The mean of the overall immersion level of Group 1 is 5, whereas Group 2 is 6 . No statistically significant differences between the two groups were found in this study.

\section{Discussion}

This study developed a system that integrates MUVR applications into omnidirectional treadmills. A prototype was set up and tested by the participants to validate the system in user interaction, user locomotion, and system synchronization. This section discusses the results of applying the system in architectural design review and collaboration.

Firstly, collaborative VR allows multiple users to attend design review sessions in the shared virtual environment. According to the results, the system can enhance interaction experiences facilitated by the interactive functions of the VR platform. Based on the means of the user interaction measures, they were rated well by the participants, especially the interactive experience during the design overview. In this case, the participants used most interaction tools (i.e., scale-model mode, measurement tool, and primitive creation tool) to review design options of the building model with their partners. With the VR treadmills, the participants could walk around the same way that they would in reality, achieving more realistic interactions and collaboration in the immersive virtual environment. Moreover, VR is not just a valuable means of design visualizations or model walkthroughs; it can also be used as an effective design tool. According to the results, designing in the virtual environment is possible. The interactive functions of the system allow the innovative design to be created in the shared virtual environment. For the conflict resolutions and design detailing, the participants were satisfied with the system. During the testing, the participants were involved in the tasks about clarifications of the headroom clearance of lift door openings and verifications of the assembly details of glass balustrades. Using MUVR for architectural design review allows architects and their team members to step into the building model for interactions. They can assess any virtual spaces inside the building to better understand spatial requirements and stimulated final products. This type of collaborative immersion enhances strong team communication and enables true collaborative design, ultimately speeding up the whole design process. These findings are in line with those of previous studies on the effects of VR on the design review process, particularly the enhanced interactions arising from MUVR applications.

Secondly, human locomotion is a VR technology that enables the movement from one place to another in the virtual environment. In this study, human locomotion was enabled by the omnidirectional treadmills that offer users high-level simulations of the natural movement and alleviate their VR sickness. The participants highly appreciated the system's safety, as the harness protection locks the user securely on the treadmills. Generally, VR applications can be set up in any space. For that reason, the larger the room space, the farther the users can walk within the perceived environment. However, for a room-scale VR setup, the users might come to the physical limits of the room and choose to teleport themselves to a different virtual location. In this study, the participants walked around the building models to review architectural design without limitations. However, the participants showed concerns about the agility and preciseness of using the system. The 
omnidirectional treadmills provide platforms that enhance the illusion of unconstrained movement. With the harness protections, the participants can move naturally in a linear direction on every building floor. Nevertheless, running motions inside the building model were not recommended, due to the slipperiness of the overshoes worn by the users. Most of the participants also felt difficulty stopping precisely, due to the reduced foot friction on the treadmills. This deficiency disturbed the participants, because they needed to move back and forth until reaching a particular location to discuss the design matter with their partners. Further, when the participants came to the staircase, the walking feature became an inclination mode in which users moved like climbing a steep ramp. In this case, the participants experienced a reduced reality level in natural walking. Interestingly, it was found that human locomotion experiences between the practitioners and students were different, particularly the walking speed and versatility of the practitioners on the treadmills. Before the testing, the practitioners took much time to learn the treadmill operations. They also showed concerns about the erratic movement when walking on the treadmills. Comparatively, the students got used to the treadmills fast, and it took a while to accustom themselves to moving on them. This kind of new technology is exciting and appealing to younger generations, and they will gravitate towards gaming applications such as the MUVR treadmill system developed in this study. Thus, the students indicated a strong intent to be engaged in the testing without hesitation. These results align with the literature on technology readiness, which advocates the influence of human age over technology use.

Thirdly, the system is the integration of VR devices into the omnidirectional treadmills with the MUVR server. As the server plays an essential role in optimizing the synchronization performance between multiusers, its performance is one of the critical factors for MUVR success. According to the results, the stability and reliability of the system were rated well by the participants. These outcomes indicate the participants' satisfaction with the synchronization speed and the ability to synchronize data between multiusers when moving and interacting in the virtual environment. Nevertheless, if model scaling was activated extensively to involve many objects in a single frame, the synchronization speed was reduced when extra latency was introduced. Most of the participants observed that the objects or mark-ups created can be synchronized well with suitable resolutions in a 1:1 ratio of the virtual environment.

The practitioners were interviewed after the testing. All of them considered that collaborative VR is a practical approach to architectural design review with inexpensive VR devices available in the market. Under the MUVR mode, architects can collaborate with their team members and adopt various intuitive tools for their interactions. With the integration of the omnidirectional treadmills, unobtrusive and natural-feeling locomotion is achieved by the system, helping users engage fully in the immersive virtual environment. In addition, the free and natural movement over the treadmills relieves motion sickness from disorienting experiences. However, there are still several critical challenges that need to be overcome. The participants addressed some limitations and suggestions of the system to enhance its practical effectiveness. First, creating a computer-generated 3D model for use in VR requires a much higher level of detail to be incorporated into this model. Every visible surface needs to be modeled, and the construction details should be fully resolved. Failure to do so will highlight design deficiencies when viewed in this artificial reality. The practitioners expected the system to be more accurate due to their rich practical experience and exposure to the real-life design review process. Second, the MUVR experience can be made more immersive by adding an appropriate audio track replicating sounds likely to be heard in the virtual environment, making the sensory experience even more realistic. Third, the locomotion setting can be improved by providing walking-speed adjustments or other customizations so that every user can be accustomed to the system expeditiously. Fourth, the enhanced graphics processing unit of the system can improve the reduced synchronization speed, due to network latency. However, this enhancement requires an immense amount of computing power to process quality computer graphics. Finally, the 
system can integrate other BIM-based 3D models (e.g., structural models and MEP models) into the current architectural model to achieve interdisciplinary design coordination.

\section{Limitations and Future Research Areas}

Several limitations to this study need to be acknowledged. Some of them are connected to inform future research areas. First, a total of eight participants joined the system testing. Although the sample size is not large, the data were collected from the participants during the in-person interview, and the whole testing setup was rigorous, to secure quality data collection. Second, the MUVR treadmill system was developed to involve a pair of participants to accomplish several design review tasks. Such multiple-user settings limit the scope of this study. The system can be enhanced for further investigation of the outcomes of individual-user participation and the VR experiences of single and multiple users in different scenarios. Third, this study was focused on architectural design review and collaboration. Thus, all participants invited are practitioners and students in the field of architecture. Further research is recommended to investigate how other construction professionals, such as engineers and surveyors, can adopt the system.

\section{Conclusions}

The BIM-based MUVR treadmill system was developed to enhance the immersion level of architectural design review and collaboration. The prototype of the system was verified and validated by eight participants. The results revealed that the system performed well in user interactions in the virtual environment. Significantly, the participants were most satisfied with the design overview function that allows architects and their team members to review the overall architectural design with increased collaboration. To enhance the immersive experience, the participants walked around and navigated the building model on the VR treadmills. This study confirmed the safety performance of the treadmills. However, the identified challenges are the reduced level of reality when walking along staircases and the inaccuracy of precise stops at any particular location. Further, as the system comprises new VR technology, the participants were divided into two groups to detect any unconscious bias from different user generations. The findings demonstrated that the group of practicing architects and the group of architecture students scored differently in the agility of VR locomotion. This result reflects the new technology readiness of the younger generation when using VR devices. This observation is the only significant difference identified in this study between the two participant groups. This study also proved that the MUVR server was stable and reliable. The system performed reasonably to synchronize data between multiple VR users. Finally, the participants recommended several suggestions to maximize the system's effectiveness, such as improving the level of detail of the VR model, providing audio tracks to users, and enhancing the computing power for graphics processing. To conclude, VR has been gradually applied in the AEC industry, and the need for BIM-based collaboration capabilities has increased. As a result, collaborative VR has been recognized as an essential application for design and construction. Thus, the system improves the level of immersion in MUVR applications and paves the way to VR innovations in response to industry needs. The system allows architects to interact directly with their team members and participate more collaboratively in the design review process.

Author Contributions: Conceptualization, C.C.W.K. and J.I.K.; methodology, C.C.W.K. and J.I.K.; software, C.C.W.K. and Q.M.O.; validation, C.C.W.K., J.I.K. and Q.M.O.; formal analysis, C.C.W.K. and Q.M.O.; data curation, Q.M.O.; writing—original draft preparation, C.C.W.K.; writing-review and editing, J.I.K.; supervision, C.C.W.K.; project administration, C.C.W.K.; funding acquisition, C.C.W.K. All authors have read and agreed to the published version of the manuscript.

Funding: This research was funded by the Teaching Development Grant (project no. 6000714) from City University of Hong Kong.

Conflicts of Interest: The authors declare no conflict of interest. 


\section{References}

1. Briscoe, D. Beyond BIM-Architecture Information Modeling; Routledge: London, UK, 2016.

2. Oh, M.; Lee, J.; Hong, S.W.; Jeong, Y. Integrated system for BIM-based collaborative design. Autom. Constr. 2015, 58, 196-206. [CrossRef]

3. Love, P.E.D.; Irani, Z.; Edwards, D.F. A seamless supply chain management model for construction. Supply Chain Manag. 2004, 9 , 43-56. [CrossRef]

4. Hsieh, M.C.; Lee, J.J. Preliminary study of VR and AR applications in medical and healthcare education. J. Nurs. Health Stud. 2017, 3, 1. [CrossRef]

5. Kim, J.I.; Li, S.; Chen, X.; Keung, C.; Suh, M.; Kim, T.W. Evaluation framework for BIM-based VR applications in design phase. J. Comput. Des. Eng. 2021, 8, 910-922.

6. Radianti, J.; Majchrzak, T.A.; Fromm, J.; Wohlgenannt, I. A systematic review of immersive virtual reality applications for higher education: Design elements, lessons learned, and research agenda. Comput. Educ. 2020, 147, 103778. [CrossRef]

7. Berg, L.P.; Vance, J.M. An industry case study: Investigating early design decision making in virtual reality. J. Comput. Inf. Sci. Eng. 2017, 17, 011001. [CrossRef]

8. Boton, C. Supporting constructability analysis meetings with Immersive Virtual Reality-based collaborative BIM 4D simulation. Autom. Constr. 2018, 96, 1-15. [CrossRef]

9. Bouchlaghem, D.; Shang, H.; Whyte, J.; Ganah, A. Visualisation in architecture, engineering and construction (AEC). Autom. Constr. 2005, 14, 287-295. [CrossRef]

10. Portman, M.E.; Natapov, A.; Fisher-Gewirtzman, D. To go where no man has gone before: Virtual reality in architecture, landscape architecture and environmental planning. Comput. Environ. Urban Syst. 2015, 54, 376-384. [CrossRef]

11. Sidani, A.; Dinis, F.M.; Sanhudo, L.; Duarte, J.; Baptista, J.S.; Martins, J.P.; Soeiro, A. Recent tools and techniques of BIM-based virtual reality: A systematic review. Arch. Comput. Methods Eng. 2021, 24, 449-462. [CrossRef]

12. Li, H.; Chan, G.; Skitmore, M. Multiuser virtual safety training system for tower crane dismantlement. J. Comput. Civ. Eng. 2012, 26, 638-647. [CrossRef]

13. Hooks, K.; Ferguson, W.; Morillo, P.; Cruz-Neira, C. Evaluating the user experience of omnidirectional VR walking simulators. Entertain. Comput. 2020, 34, 100352. [CrossRef]

14. Chang, E.; Kim, H.T.; Yoo, B. Virtual reality sickness: A review of causes and measurements. Int. J. Hum. -Comput. Interact. 2020, 36, 1658-1682. [CrossRef]

15. Regenbrecht, H.; Donath, D. Architectural education and virtual reality aided design (VRAD). In Designing Digital Space-An Architect's Guide to Virtual Reality; Bertol, D., Ed.; John Wiley and Sons: New York, NY, USA, 1997.

16. Boman, D.K. International survey: Virtual-environment research. Constr. Build. Mater. 1994, 8, 70-71. [CrossRef]

17. Sherman, B.; Judkins, P. Glimpses of Heaven, Visions of Hell: Virtual Reality and its Implications; Hodder and Stoughton: London, UK, 1992.

18. Dunston, P.S.; Arns, L.L.; Mcglothlin, J.D.; Lasker, G.C.; Kushner, A.G. An immersive virtual reality mock-up for design review of hospital patient rooms. In Collaborative Design in Virtual Environments; Wang, X., Tsai, J.J.-H., Eds.; Springer: Chennai, India, 2011; pp. 167-176.

19. Allen, R.C.; Singer, M.J.; McDonald, P.J.; Cotton, J.E. Age differences in a virtual reality entertainment environment: A field study. In Proceedings of the IEA 2000/HFES 2000 Congress, San Diego, CA, USA, 30 July-4 August 2000; pp. 542-545.

20. Tsourela, M.; Roumeliotis, M. The moderating role of technology readiness, gender, and sex in consumer acceptance and actual use of technology-based services. J. High Technol. Manag. Res. 2015, 26, 124-136. [CrossRef]

21. Parasuraman, A. Technology readiness index (TRI) a multiple-item scale to measure readiness to embrace new technologies. $J$. Serv. Res. 2000, 2, 307-320. [CrossRef]

22. Eadie, R.; Browne, M.; Odeyinka, H.; McKeown, C.; McNiff, S. BIM implementation throughout the UK construction project lifecycle: An analysis. Autom. Constr. 2013, 36, 145-151. [CrossRef]

23. Wang, P.; Wu, P.; Wang, J.; Chi, H.L.; Wang, X. A critical review of the use of virtual reality in construction engineering education and training. Int. J. Environ. Res. Public Health 2018, 15, 1204. [CrossRef] [PubMed]

24. Mehrbod, S.; Staub-French, S.; Mahyar, N.; Tory, M. Beyond the clash: Investigating BIM-based building design coordination issue representation and resolution. J. Inf. Technol. Constr. 2019, 24, 33-57.

25. Zaker, R.; Coloma, E. Virtual reality-integrated workflow in BIM-enabled projects collaboration and design review: A case study. Vis. Eng. 2018, 6, 4. [CrossRef]

26. Maldovan, K.D.; Messner, J.I.; Pe, M.F. Frameworks for reviewing mock-ups in an immersive environment. In Proceedings of the 6th International Conference on Construction Applications of Virtual Reality (CONVR 2006), Orlando, FL, USA, 3-4 August 2006.

27. Sharma, S.; Chen, W. Multi-user VR classroom with 3D interaction and real-time motion detection. In Proceedings of the 2014 International Conference on Computational Science and Computational Intelligence (CSCI 2014), IEEE Computer Society, Las Vegas, NV, USA, 10-13 March 2014; pp. 187-192.

28. Shattuck, D.W. Multiuser virtual reality environment for visualising neuroimaging data. Healthc. Technol. Lett. 2018, 5, 183-188. [CrossRef] [PubMed]

29. Shi, Y.; Du, J.; Lavy, S.; Zhao, D. A multiuser shared virtual environment for facility management. Procedia Eng. 2016, 145, 120-127. [CrossRef] 
30. Hilfert, T.; König, M. Low-cost virtual reality environment for engineering and construction. Vis. Eng. 2016, 4. [CrossRef]

31. Kreimeier, J.; Karg, P.; Götzelmann, T. BlindWalkVR: Formative insights into blind and visually impaired people's VR locomotion using commercially available approaches. In Proceedings of the 13th Pervasive Technologies Related to Assistive Environments Conference (PETRA'20), Corfu, Greece, 30 June-3 July 2020.

32. Sloot, L.H.; Krogt, M.M.; Harlaar, J. Effects of adding a virtual reality environment to different modes of treadmill walking. Gait Posture 2014, 39, 939-945. [CrossRef]

33. Jochymczyk-Woźniak, K.; Nowakowska, K.; Polechonski, J.; Sladczyk, S.; Michnik, R. Physiological gait versus gait in VR on multidirectional treadmill-Comparative analysis. Medicina 2019, 55, 517. [CrossRef]

34. Darken, R.P.; Cockayne, W.R.; Carmein, D. The omni-directional treadmill: A locomotion device for virtual worlds. In Proceedings of the 10th Annual Symposium on User Interface Software and Technology (UIST 97), Banff, AB, Canada, 14-17 October 1997; pp. 213-221.

35. Dęskka, M.; Polechonski, J.; Mynarski, A.; Polechonski, P. Enjoyment and intensity of physical activity in immersive virtual reality performed on innovative training devices in compliance with recommendations for health. Int. J. Environ. Res. Public Health 2019, 16, 3673. [CrossRef]

36. Davidson, J.; Fowler, J.; Pantazis, C.; Sannino, M.; Walker, J.; Sheikhkhoshkar, M.; Rahimian, F.P. Integration of VR with BIM to facilitate real-time creation of bill of quantities during the design phase: A proof of concept study. Front. Eng. Manag. 2019, 7, 396-403. [CrossRef]

37. Froehlich, M.A.; Azhar, S. Investigating virtual reality headset applications in construction. In Proceedings of the 52nd ASC Annual International Conference, Brigham Young University, Provo, UT, USA, 13-16 April 2016.

38. Wang, C.; Li, H.; Kho, S.Y. VR-embedded BIM immersive system for QS engineering education. Comput. Appl. Eng. Educ. 2018, 26, 626-641. [CrossRef]

39. Schofield, D.; Cox, C.J.B. The use of virtual environments for percentage view analysis. J. Environ. Manag. 2005, 76, 342-354. [CrossRef] [PubMed]

40. Dickson, P.E.; Block, J.E.; Echevarria, G.N.; Keenan, K.C. An experience-based comparison of unity and unreal for a stand-alone 3D game development course. In Proceedings of the Annual Conference on Innovation and Technology in Computer Science Education, ITiCSE, Bologna, Italy, 3-5 July 2017; pp. 70-75.

41. Suznjevic, M.; Mandurov, M.; Matijasevic, M. Performance and QoE assessment of HTC Vive and Oculus Rift for pick-and-place tasks in VR. In Proceedings of the 9th IEEE International Conference on Quality of Multimedia Experience (QoMEX 2017), Erfurt, Germany, 31 May-2 June 2017; pp. 3-5.

42. Funkhouser, T.A. Ring: A client-server system for multi-user virtual environments. In Proceedings of the Symposium on Interactive 3D Graphics (SI3D'95), Monterey, CA, USA, 9-12 April 1995; pp. 85-92.

43. Du, J.; Zou, Z.; Shi, Y.; Zhao, D. Zero latency: Real-time synchronization of BIM data in virtual reality for collaborative decision-making. Autom. Constr. 2018, 85, 51-64. [CrossRef]

44. Hair, J.F.; Anderson, R.E.; Tatham, R.L.; Black, W.C. Multivariate Data Analysis, 5th ed.; Patience Hall: Upper Saddle River, NJ, USA, 1998. 